\title{
Acute Pancreatitis in a Patient with Subarachnoid Hemorrhage: A Causal Link or a Mere Coincidence?
}

\author{
Amit Goyal ${ }^{1}$, Mathangi Krishnakumar², Muthuchellapan Radhakrishnan ${ }^{3}$, Dwarakanath Srinivas ${ }^{4}$
}

\begin{abstract}
Both subarachnoid hemorrhage (SAH) and acute pancreatitis (AP) are associated with systemic inflammatory response leading to extracerebral multiple organ dysfunction. In this case report, we describe an adult male, who developed AP in the postoperative period following surgical clipping of an anterior communicating artery aneurysm. The diagnosis of pancreatitis got delayed because the patient showed signs of systemic inflammation which would also been seen following SAH.

Keywords: Encephalopathy, Meningitis, Pancreatitis, Sepsis, Subarachnoid hemorrhage.

Indian Journal of Critical Care Medicine (2020): 10.5005/jp-journals-10071-23348
\end{abstract}

\section{INTRODUCTION}

Subarachnoid hemorrhage (SAH) following rupture of an intracranial aneurysm causes a sudden rise in intracranial pressure which triggers the sympathetic nervous system and endocrine hormonal responses. This results in extracerebral multiple organ dysfunction due to generalized inflammatory response and heightened sympathetic activity. Manifestations of this phenomenon include left ventricular contractile dysfunction, pulmonary edema, pneumonia, hyperglycemia, and dyselectrolytemia. ${ }^{1}$ Acute pancreatitis (AP), an inflammatory disease of the pancreas, also presents with systemic inflammation involving multiple organs. ${ }^{2}$ It is usually a complication of alcohol abuse or biliary tract disorders. However, it has also been described in patients with neurologic injury like status epilepticus. ${ }^{3}$ Herein, we report a case of AP detected postoperatively in a patient who underwent clipping of anterior communicating artery aneurysm.

\section{Case Description}

A 47-year-old man presented with complaints of persistent, severe headache of one and a half months duration. He had no known comorbid illness. He was a chronic smoker and nonalcoholic. Computed tomographic (CT) imaging of the head showed diffuse bleed in the subarachnoid space extending into the ventricles (Fisher grade IV). He had no neurological deficits (World Federation of Neurological Surgeons grade I). Digital subtraction angiography (DSA) of the cerebral vessels showed an anterior communicating artery aneurysm measuring $1.6 \times 4.0 \times 6.3 \mathrm{~mm}$. His routine blood investigations, electrocardiogram, and echocardiogram at admission were unremarkable. On the evening of his admission to the hospital, he deteriorated neurologically and was showing flexor response to painful stimuli. Computed tomographic imaging of the head showed a fresh bleed along with hydrocephalus requiring an external ventricular drain (EVD). Following EVD, his sensorium improved and was taken up for emergency surgery to isolate the aneurysm from the parent vessel with permanent clips and for converting his EVD to ventriculoperitoneal (VP) shunt. Following surgery, he was electively ventilated for 24 hours and then extubated. He was neurologically well preserved [Glasgow Coma Scale (GCS) E3M6V5] and was shifted to the ward. Two days later,

\begin{abstract}
${ }^{1-3}$ Department of Neuroanesthesia and Neurocritical Care, National Institute of Mental Health and Neurosciences, Bengaluru, Karnataka, India

${ }^{4}$ Department of Neurosurgery, National Institute of Mental Health and Neurosciences, Bengaluru, Karnataka, India

Corresponding Author: Amit Goyal, Department of Neuroanesthesia and Neurocritical Care, National Institute of Mental Health and Neurosciences, Bengaluru, Karnataka, India, Phone: +91 8800801784 , e-mail: amitgoyal26@ymail.com
\end{abstract}

How to cite this article: Goyal A, Krishnakumar M, Radhakrishnan M, Srinivas D. Acute Pancreatitis in a Patient with Subarachnoid Hemorrhage: A Causal Link or a Mere Coincidence? Indian J Crit Care Med 2020;24(2):141-142.

Source of support: Departmental Resources

Conflict of interest: None

he developed fever, hypotension, and deterioration in sensorium with GCS E2M5V2. Sepsis was suspected, and he was resuscitated with fluids, antibiotics, elective ventilation, and a noradrenaline infusion to keep up his blood pressure keeping vasospasm in mind. A repeat DSA of the cerebral vessels showed complete clipping of the aneurysm without any evidence of vasospasm. Laboratory tests for identifying the source of sepsis did not yield any positive results. Two days following his intensive care unit admission, he developed abdominal distension with persisting fever. An ultrasound examination of the abdomen showed moderate ascites and bilateral pleural effusion, both of which did not require tapping. He remained neurologically stable with localizing pain to painful stimuli and opening eyes to call. He developed severe hypoalbuminemia (serum albumin $1.53 \mathrm{~g} / \mathrm{dL}$ ) for which intravenous albumin was administered. His abdominal distension was interfering with this respiratory efforts and he failed to wean from the ventilator. During workup for his abdominal distension, we observed an elevated lipase $1830 \mathrm{U} / \mathrm{L}$ (laboratory baseline upper limit value: 170 $\mathrm{U} / \mathrm{L}$ ) with normal serum amylase, calcium, and triglyceride levels. Suspecting AP, CT abdomen was ordered which showed edematous pancreas with peripancreatic fat stranding (Fig. 1). He was managed conservatively with antibiotics, antipyretics, and total parental nutrition. A week later, he showed fluctuations in his sensorium. 


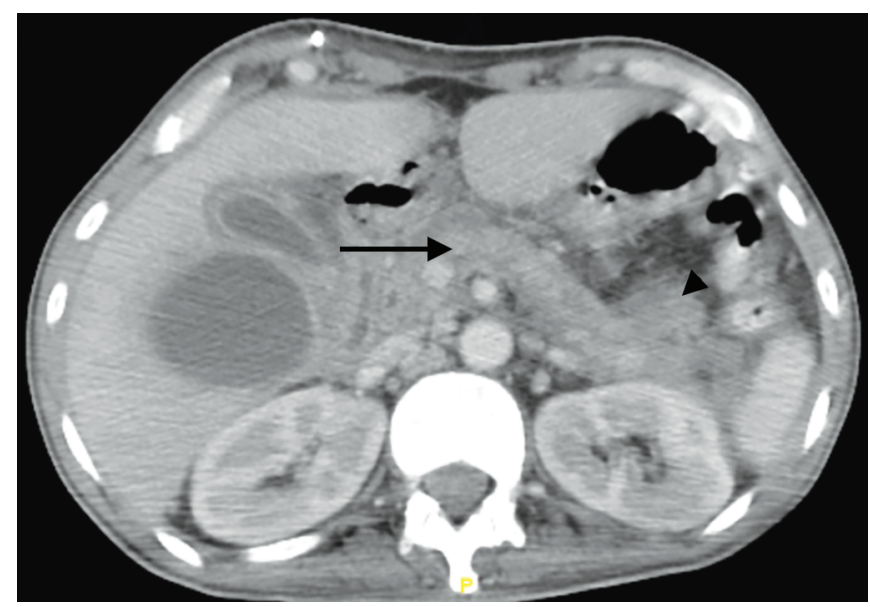

Fig. 1: Axial contrast-enhanced computed tomographic abdomen showing heterogeneously enhancing pancreas with a bulky pancreatic tail (arrow) and peripancreatic fat stranding (arrow head)

Suspecting inadequate cerebrospinal fluid (CSF) drainage due to high abdominal pressure, externalization of VP shunt was done which improved his neurologic status. Laboratory testing of ascitic fluid (collected at the time of externalization) showed no signs of infection. Two weeks later, when his abdominal distension began to subside, he was tracheostomized and weaned from the ventilator. After further 2 weeks of observation, his GCS improved to E4M6Vt, enteral feedings initiated, and his lipase level returned to baseline values and was shifted to the ward. After spending 2 weeks in the ward, he again developed fever and altered sensorium. Redness, induration, purulent discharge, and subcutaneous swelling were noted at the abdominal entry point of the shunt tube. The purulent discharge, tracheal secretions, and CSF grew Klebsiella, and he was put on appropriate antibiotics. However, he went into septic shock, refractory to treatment and died a week later.

\section{Discussion}

Acute pancreatitis in a patient with $\mathrm{SAH}$ has not been reported. Both SAH and AP induce severe systemic inflammatory response, and it is difficult to comment whether AP onset was sporadic or was a consequence of SAH. Since both disease processes manifest symptoms of systemic inflammatory response syndrome (SIRS) and patient was unable to communicate about the abdominal pain (due to altered sensorium and endotracheal intubation), diagnosis of AP got delayed in this patient. Ascites and pleural effusion were suspected to be due to hypoalbuminemia and AP was not considered early as this patient did not carry any risk factors. Our patient had an isolated increase in serum lipase with normal serum amylase. As amylase has a shorter half-life than lipase, it might have returned to normal levels before the diagnosis was made. However, serum lipase has superior diagnostic accuracy when compared with amylase. ${ }^{4}$ Although the incidence is very low, few anticonvulsants have been associated with AP such as valproic acid and carbamazepine., ${ }^{5,6}$ Our patient was receiving phenytoin, which is not known to cause pancreatic injury.

Absence of known etiological factors, suggest that AP might have occurred secondary to $\mathrm{SAH}$, and deterioration in sensorium could have been additionally contributed by pancreatic encephalopathy which manifests in the early stage of AP. ${ }^{7-9}$ Likely mechanisms of pancreatic injury in SAH include oxidative stress and SIRS. ${ }^{10}$

After $\mathrm{SAH}$, cardiopulmonary and renal complications get early attention, and gastrointestinal complications remain unnoticed. Delayed unsuspected diagnosis of AP can be fatal. Although AP was managed successfully, the patient succumbed to meningitis which could have spread from the abdomen through the shunt tube path.

This case report highlights the fact that AP can occur as one of the extracerebral organ dysfunction following $\mathrm{SAH}$ and should be considered early in the differential diagnosis in SAH patients who develop abdominal distension.

A written consent for publication of this case has been obtained from the patient's kin.

\section{References}

1. Chen S, Li Q, Wu H, Krafft PR, Wang Z, Zhang JH. The harmful effects of subarachnoid hemorrhage on extracerebral organs. Biomed Res Int 2014;2014:858496. DOI: 10.1155/2014/858496.

2. Cappell MS. Acute pancreatitis: etiology, clinical presentation, diagnosis, and therapy. Med Clin North Am 2008;92(4):889-923. DOI: 10.1016/j.mcna.2008.04.013.

3. Ogawa K, Kanemoto K, Shirasaka Y, Kawasaki J, Yamasaki S. Acute pancreatic damage associated with convulsive status epilepticus: a report of three cases. Psychiatry Clin Neurosci 2001;55(6):619-622. DOI: 10.1046/j.1440-1819.2001.00914.x.

4. Working Party of the British Society of Gastroenterology, Association of Surgeons of Great Britain and Ireland, Pancreatic Society of Great Britain and Ireland, Association of Upper GI Surgeons of Great Britain and Ireland. UK guidelines for the management of acute pancreatitis. Gut 2005;54(Suppl 3):iii1-iii9. DOI: 10.1136/gut.2004.057026.

5. Moreiras Plaza M, Rodriguez Goyanes G, Cuina L, Alonso R. On the toxicity of valproic-acid. Clin Nephrol 1999;51(3):187-189.

6. Forte A, Gallinaro L, Montesano G, Turano R, Bertagni A, Illuminati G. A possible case of carbamazepine induced pancreatitis. Riv Eur Sci Med Pharmacol 1996;18(5-6):187-189.

7. Zhang $X P$, Tian $H$. Pathogenesis of pancreatic encephalopathy in severe acute pancreatitis. Hepatobiliary Pancreat Dis Int 2007;6(2):134-140.

8. Weathers AL, Lewis SL. Rare and unusual... or are they? Less commonly diagnosed encephalopathies associated with systemic disease. Semin Neurol 2009;29(2):136-153. DOI: 10.1055/s-00291213734.

9. Sharf B, Bental E. Pancreatic encephalopathy. J Neurol Neurosurg Psychiatry 1971;34:357-361.

10. Rau B, Poch B, Gansauge F, Bauer A, Nüssler AK, Nevalainen $T$, et al. Pathophysiologic role of oxygen free radicals in acute pancreatitis: initiating event or mediator of tissue damage? Ann Surg 2000;231(3):352-360. DOI: 10.1097/00000658-200003000-00008. 